1 Ministério da Saúde (MS)

- Brasília (DF), Brasil.

claudia.brandao@saude.

gov.br

2 Universidade de Brasília (UnB) - Brasília (DF),

Brasil.

3 Universidade Federal da Bahia (UFBA), Instituto de Saúde Coletiva (ISC) Salvador (BA), Brasil.

4 Universidade do Estado do Rio de Janeiro (Uerj), Instituto de Medicina Social (IMS), Grupo de Pesquisa Políticas, Programas e Ações de Educação na Saúde (PPAES) - Rio de Janeiro (RJ), Brasil.

5 Universidade Federal da Bahia (UFBA), Instituto de Humanidades, Artes e Ciências - Salvador (BA) Brasil.

\section{A retomada do processo de implementação da Política Nacional de Educação Permanente em Saúde no Brasil}

\author{
The resumption of the implementation process of the National \\ Permanent Health Education Policy in Brazil
}

Cláudia Brandão Gonçalves $\mathbf{1 , 2}$, Isabela Cardoso de Matos Pinto $\mathbf{3}$, Tania França ${ }^{\mathbf{4}}$, Carmen Fontes Teixeira ${ }^{5}$

DOI: 10.1590/0103-11042019S101

RESUMO Este trabalho teve por objetivos descrever e analisar as mais recentes iniciativas realizadas, no âmbito da gestão federal, para a implementação e fortalecimento da Política Nacional de Educação Permanente em Saúde (PNEPS). Toma como referencial o ciclo da política pública de Kingdon, descrevendo como se deu a inclusão desse tema na agenda governamental, em 2003, e analisa o conteúdo dos documentos que materializam as propostas da Política. Em seguida, concentra-se na descrição e análise do movimento desencadeado em 2017-2018, com a realização de uma série de eventos regionais, dos quais resultaram a identificação das fragilidades enfrentadas nesse processo de implementação da PNEPS, subsidiando um conjunto de propostas, que referenciam as iniciativas desencadeadas, visando à efetiva implementação da PNEPS no âmbito do Sistema Único de Saúde.

PALAVRAS-CHAVE Política pública. Educação continuada. Sistema Único de Saúde. Recursos humanos.

ABSTRACT This work aimed to describe and to analyze the most recent initiatives carried out, within the scope of federal management, for the implementation of the National Permanent Health Education Policy (PNEPS). It takes as reference the Kingdon public policy cycle, describing how the topic was included in the government agenda in 2003, and analyzes the content of the documents that materialize the proposals of the Policy. Subsequently, it focuses on the description and analysis of the movement triggered in 2017-2018, with the accomplishment of a series of regional events, which resulted in the identification of the weaknesses faced in the implementation process of the PNEPS, subsidizing a set of proposals that referenced the initiatives triggered, aiming at the effective implementation of the PNEPS in the scope of the Unified Health System (SUS).

KEYWORDS Public policy. Education continuing. Unified Health System. Human resources. 


\section{Introdução}

O debate internacional desenvolvido atualmente em torno das questões relativas ao trabalho e educação na saúde abrange a reflexão sobre políticas, regulamentações e intervenções relacionadas com a educação, capacitação e práticas profissionais, destacando-se a necessidade de articular a capacitação com as competências requeridas pelo trabalho, a utilização de novas metodologias de ensino e a incorporação de tecnologias de informação, educação e comunicação em saúde'.

Esse tema aparece entre os compromissos assumidos pelos países perante a agenda $2030^{1}$ como parte das estratégias de fortalecimento dos sistemas universais de saúde, compromisso reiterado, em 2017, na Conferência Panamericana de Saúde 2 , quando se discutiu a situação dos recursos humanos em saúde no continente, apontando-se desafios recorrentes, tais como: inadequação dos perfis profissionais, precariedade das condições de trabalho, baixa produtividade e limitada qualidade do desempenho, entre outros.

No caso do Brasil, cabe reconhecer que o processo de construção do Sistema Único de Saúde (SUS) ${ }^{3}$, ao longo dos últimos 30 anos, contemplou a implementação de políticas e programas que resultaram na descentralização da gestão, ampliação de cobertura das ações e reorganização dos serviços de saúde 4 , tendo como uma das consequências a reconfiguração do mercado de trabalho no setor e o considerável aumento da força de trabalho direta ou indiretamente vinculada à produção de ações e serviços de saúde em vários níveis de complexidade ${ }^{5}$.

Esse processo tem colocado a necessidade de promover mudanças/transformações na formação de pessoal, quer no âmbito dos cursos de graduação e pós-graduação, quer no âmbito dos serviços, por meio de ações de educação permanente, o que acentuou a preocupação com as relações entre as instituições que compõem o sistema de formação de pessoal e o sistema de saúde 5,6, tema que desafia estudiosos da área, gestores e profissionais de saúde.
Nesse sentido, a criação, em 2003, da Secretaria de Gestão do Trabalho e Educação na Saúde (SGTES) do Ministério da Saúde (MS) permitiu a movimentação de ideias, conhecimentos, programas e recursos financeiros, ampliando as possibilidades de desenvolvimento de políticas governamentais específicas nessa área, além de estimular o diálogo entre diferentes atores de instituições parceiras e entidades representativas da gestão do SUS, como o Conselho Nacional de Secretários de Saúde (Conass), o Conselho Nacional de Secretários Municipais de Saúde (Conasems) e o Conselho Nacional de Saúde (CNS).

A atuação da SGTES contemplou, entre outras iniciativas relevantes, a formulação da Política Nacional de Educação Permanente em Saúde (PNEPS), lançada em 2003, e institucionalizada com a publicação da Portaria GM/ $\mathrm{MS} \mathrm{n}^{0} 198$, de 13 de fevereiro de $2004^{7}$, que estabeleceu diretrizes para sua implementação, fomentando a condução regional da política e a participação interinstitucional e intersetorial. Posteriormente, foi publicada a Portaria GM/ MS 1.996/078, a qual propôs novas diretrizes para a PNEPS, que integram, atualmente, a base normativa do SUS, consolidada em $2017^{9}$.

A concepção de Educação Permanente adotada nesse marco normativo considera que esta implica o estabelecimento de

[...] relações orgânicas entre ensino e as ações e serviços, e entre docência e atenção à saúde bem como as relações entre formação e gestão setorial, desenvolvimento institucional e controle social em saúde ${ }^{8(34)}$.

Destina-se, assim, a públicos multiprofissionais, isto é, à equipe de saúde inserida nos vários níveis organizacionais da rede de serviços, objetivando transformações das práticas técnicas e sociais de saúde, tendo em vista a garantia do acesso, a melhoria da qualidade, a humanização da atenção à saúde da população e o aperfeiçoamento da capacidade de gestão do SUS.

Do ponto de vista pedagógico, a Educação 
Permanente em Saúde (EPS) toma como ponto de partida para as ações educativas,

[...] os problemas identificados no processo de trabalho, utilizando metodologias ativas de ensino-aprendizagem, com ênfase na resolução de problemas, geralmente por meio de supervisão dialogada e oficinas de trabaIho realizadas, preferencialmente, no próprio ambiente de trabalho, de modo a sensibilizar e gerar compromissos entre os trabalhadores, gestores, instituições de ensino e usuários para com o desenvolvimento institucional do SUS, a melhoria do desempenho das equipes de saúde e o desenvolvimento individual dos profissionais e trabalhadores de saúde8(34).

Deve ser considerada, portanto,

[...] um processo contínuo, articulado à descentralização da gestão do sistema e à reorganização da rede de serviços, em bases territoriais, fomentando, assim, a condução regional da política, com participação interinstitucional através das Comissões de Integração Ensino Serviço8(34).

A formulação e a implementação da PNEPS estimularam a produção científica sobre esse tema, tendo sido objeto de análise em alguns estudos que apontam evidências importantes das dificuldades de articulação entre gestores, trabalhadores, instâncias de controle social e Instituições de Ensino Superior (IES), participação incipiente dos gestores municipais e dificuldades na utilização dos recursos financeiros, que repercutem na reduzida implantação das Comissões de Integração Ensino e Serviço (Cies), previstas na PNEPS, na indefinição de parâmetros para construção de projetos e na ausência de avaliação das ações executadas no que tange a mudanças nas práticas de formação, gestão e atenção na saúde 10-14 .

No momento atual, considerando-se os problemas que incidem sobre a efetiva consolidação do SUS - a exemplo do subfinanciamento ${ }^{15}$, recomposição das relações entre público e privado ${ }^{16,17}$, mudanças na gestão de unidades complexas e reorientação de políticas e estratégias prioritárias -, constata-se a necessidade de ajustes em vários âmbitos da gestão do trabalho e da educação em saúde. No que se refere especificamente à PNEPS, identificou-se a necessidade de uma reflexão acerca das estratégias a serem adotadas para garantia de sua efetiva implementação, levando-se em conta as especificidades regionais, estaduais e locais.

Nessa perspectiva, o MS, por meio do Departamento de Gestão da Educação na Saúde (Deges), vinculado à SGTES, em parceria com o Conass, Conasems, CNS, Escolas Técnicas do SUS (Retsus), Escolas de Saúde Pública (ESP), IES, Organização Pan-Americana da Saúde (Opas) e demais secretarias do MS, deu início ao processo de discussão sobre a PNEPS com o objetivo de debater coletivamente estratégias para atualizar a Portaria GM/MS n ${ }^{0} 1.996 / 07$.

Assim, acordou-se a realização de Oficinas Regionais, envolvendo estados e municípios, para avaliação do processo de implementação da Política, cuja organização foi construída coletivamente envolvendo o Deges/SGTES com a cooperação técnica das equipes do Instituto de Saúde Coletiva da Universidade Federal da Bahia (ISC/UFBA), do Instituto de Medicina Social da Universidade do Estado do Rio de Janeiro (IMS/Uerj), Faculdade de Medicina da Universidade Federal de Minas Gerais (UFMG), Fundação Oswaldo Cruz (Fiocruz), e a participação do Conass e do Conasems.

Foram realizadas seis Oficinas, reunindo gestores e técnicos das regiões Nordeste, Norte, Centro-Oeste, Sudeste e Sul, configurando-se um movimento de retomada do debate em torno da PNEPS, constituindo o ponto de partida para a elaboração de propostas que geraram iniciativas no âmbito do MS, bem como nos estados. Considerando a riqueza e importância estratégica desse processo, os objetivos deste artigo são descrever e analisar as atividades realizadas, bem como sistematizar as propostas elaboradas coletivamente, tendo em vista contribuir para o debate em torno da efetiva implementação da PNEPS no âmbito do SUS. 


\section{Procedimentos teórico- metodológicos}

A delimitação do objeto de estudo tomou como referencial a teoria do ciclo da política pública, desenvolvida por Kingdon ${ }^{18}$, que compreende quatro momentos distintos: a) a determinação da agenda; b) a formulação da política (identificação dos problemas, seleção de propostas e alternativas, negociação e, formalização em lei); c) a implementação da política que inclui a elaboração de planos, programas e projetos no âmbito da burocracia pública e sua execução; e d) a avaliação da política, que contempla a identificação e avaliação do processo, resultados e impacto alcançado com a implementação dos planos, programas e projetos, base para a elaboração de propostas de aperfeiçoamento e/ou mudanças nos objetivos e ações propostas no âmbito da política ${ }^{17}$.
No caso especifico da PNEPS, considerou-se que a entrada na agenda e a formulação da Política se materializaram na publicação das portarias referidas anteriormente, de modo que delimitamos como objeto de estudo as ações realizadas pelo MS em 2017-2018, sob coordenação do Deges/SGTES, para retomar e intensificar o apoio aos estados e municípios para o desenvolvimento da implementação da PNEPS. Cabe ressaltar que a implementação é o momento crucial do ciclo de uma política, na medida em que consiste na materialização das propostas em ações gerenciais e operacionais que incidem sobre os problemas identificados, ações estas realizadas pelos operadores da política, sejam gestores e técnicos responsáveis pelo planejamento e programação das ações, sejam os profissionais e trabalhadores que atuam diretamente na execução das ações ${ }^{19}$.

Figura 1. Modelo teórico de análise da implementação da Política Nacional de Educação Permanente em Saúde

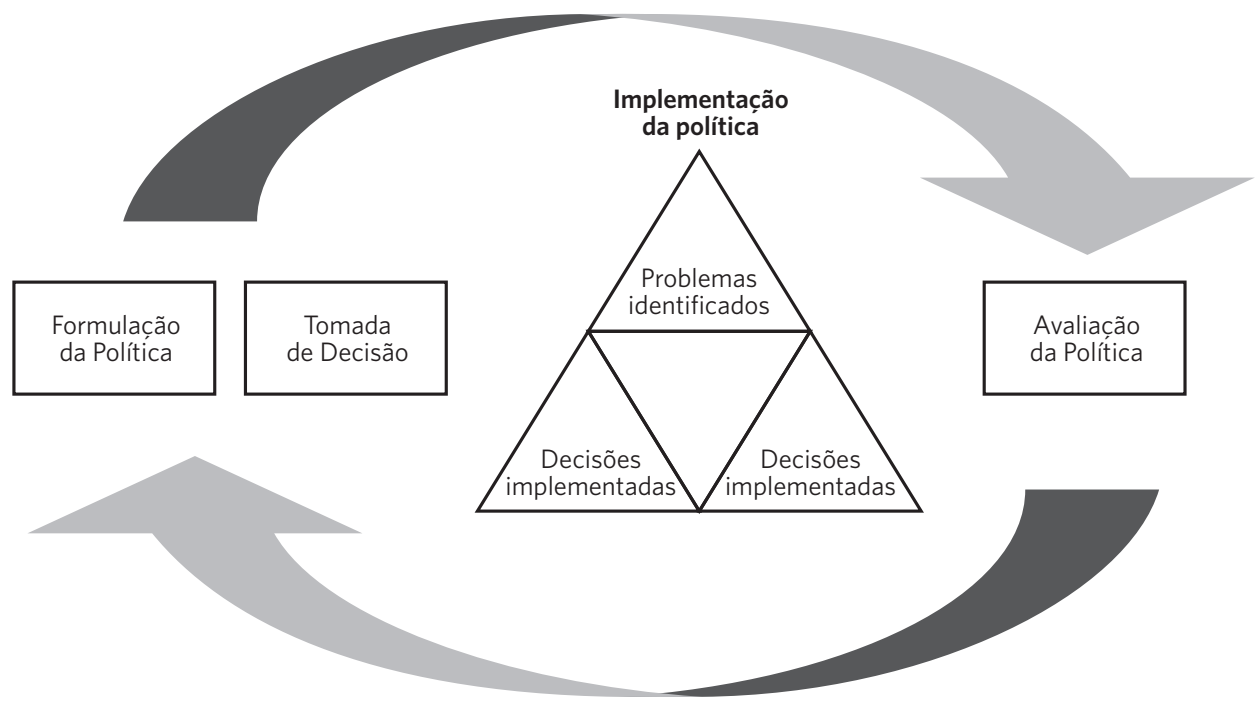

Fonte: Pinto et al.19 
As informações relativas ao processo desencadeado em 2017-2018 foram extraídas de documentos institucionais, relatórios, portarias e outras publicações do MS que registram as atividades realizadas para mobilização dos atores institucionais responsáveis pela implementação da PNEPS nos estados, tratando-se de sistematizar os resultados da análise coletiva do processo de implementação da PNEPS, as propostas, sugestões e recomendações que emanaram das Oficinas realizadas em 2017 (quadro 1) e as ações desencadeadas em 2018 para dar seguimento e concretude ao processo de implementação e avaliação da PNEPS em todo o País.

Quadro 1. Oficinas Regionais de análise da implementação da PNEPS

\begin{tabular}{|c|c|c|c|}
\hline Oficina & Data de realização & Estados Participantes & Local \\
\hline Região Nordeste & $5 / 6$ de outubro & $\begin{array}{l}\text { Bahia, Alagoas, Sergipe, Ceará, Rio } \\
\text { Grande do Norte, Piauí Pernambuco, } \\
\text { Paraíba, Maranhão }\end{array}$ & Salvador/BA \\
\hline Região Norte 1 & 19/20 de outubro & Amazônia, Pará, Acre, Rondônia & Belém/PA \\
\hline Região Norte 2 & 9/10 de novembro & Roraima, Amapá e Tocantins & Palmas/TO \\
\hline $\begin{array}{l}\text { Região Centro- } \\
\text {-Oeste }\end{array}$ & 23/24 de novembro & $\begin{array}{l}\text { Distrito Federal, Goiás, Mato Grosso, } \\
\text { Mato Grosso do Sul }\end{array}$ & Brasília/DF \\
\hline Região Sudeste & 30 de novembro e 10 de dezembro & $\begin{array}{l}\text { Espírito Santo, Minas Gerais, Rio de } \\
\text { Janeiro e São Paulo }\end{array}$ & $\begin{array}{l}\text { Belo Horizonte/ } \\
\text { MG }\end{array}$ \\
\hline Região Sul & 4/5 de dezembro & $\begin{array}{l}\text { Paraná, Santa Catarina e Rio Grande } \\
\text { do Sul }\end{array}$ & Florianópolis/SC \\
\hline
\end{tabular}

Fonte: Ministério da Saúde 22

\section{Análise do processo de formulação e implementação da PNEPS}

Como referido anteriormente, a criação da PNEPS tem como marco normativo a publicação da Portaria GM/MS no 198, de 13 de fevereiro de 2004, tendo suas diretrizes de implementação publicadas na Portaria GM/ MS n ${ }^{0}$ 1.996/07. Esses documentos contêm os objetivos e diretrizes dessa política, estabelecendo, também, as funções e responsabilidades de cada instância de gestão do SUS com relação ao desenvolvimento de ações de EPS dos profissionais e trabalhadores de saúde.

A análise desses documentos evidencia que a inclusão desse tema na agenda governamental na área de saúde constituiu um grande avanço, inserido nos esforços para o cumprimento do disposto na Lei $\mathrm{n}^{\circ} 8.080 / 90^{20}$ que explicita a responsabilidade do SUS de orientar a formação de 'recursos humanos'. As propostas contidas na PNEPS fomentam a condução regional da política, a participação interinstitucional por meio das Cies, além de definir orçamento para projetos e ações estabelecendo critérios de alocação transparentes, coerentemente, portanto, com os princípios e diretrizes gerais do SUS com relação à descentralização e participação e controle social na gestão e execução das políticas de saúde.

Além disso, cabe destacar que o processo de discussão em torno da formulação da PNEPS envolveu as instâncias de deliberação do SUS, como a Comissão Intergestores 
Tripartite (CIT), a Câmara Técnica de Recursos Humanos do Conass, a Comissão Intersetorial de Recursos Humanos e Relações de Trabalho (CIRHRT), vinculada ao CNS, assim como o Conasems.

O processo de implementação da PNEPS, por sua vez, enfrentou dificuldades, apontadas pelos estudos citados anteriormente, sendo que, na visão dos gestores e técnicos que participaram das Oficinas Regionais realizadas em 2017, esse processo ficou um tanto quanto 'adormecido', na maioria dos estados, por conta, particularmente, da interrupção do repasse de recursos financeiros do nível central (MS) para a Secretaria Estadual de Saúde (SES) a partir de 2012. Desse modo, as SES, em sua grande maioria, enfrentaram dificuldades para dar seguimento ao planejamento, programação e execução de ações de EPS, exceto nos estados onde houve investimento nessa área com recursos do orçamento estadual e/ou municipal21.

A retomada do debate sobre esse processo nas Oficinas Regionais teve como objetivos: a) identificar os principais problemas enfrentados no âmbito estadual e municipal para a implementação da PNEPS; b) identificar nós críticos que demandem apoio institucional da Deges/SGTES/ MS; c) elaborar propostas para a aperfeiçoamento da PNEPS com base nas necessidades identificadas ao nível estadual e municipal22.

A metodologia utilizada nas Oficinas possibilitou aos participantes, por meio de análises e debates, reconstruir uma imagem compartilhada da realidade em que opera a PNEPS em seus territórios. Foi possível ainda mapear as relações institucionais e uma reflexão acerca das práticas, complexidades, problemas, fortalezas, processos de gestão e de métodos pedagógicos da EPS, segundo suas próprias e singulares necessidades e possibilidades.

Cabe destacar que, para além dos seus propósitos iniciais, essas Oficinas foram vivenciadas e percebidas como uma atividade de EPS, ou seja, tornaram-se um momento de aprendizagem significativa para os participantes, constituindo-se em importante instrumento de monitoramento e acompanhamento, uma vez que permitiram examinar o desenvolvimento, os instrumentos utilizados para a gestão, os problemas, os objetivos e os resultados alcançados na sua implementação nos estados, sob a ótica das bases conceituais e organizacionais da PNEPS.

No que se refere ao conteúdo do debate travado no interior das Oficinas, cabe enfatizar, em primeiro lugar, a problematização da pertinência da revisão do marco regulatório da PNEPS, considerando-se que os princípios e diretrizes da Política continuam válidos, bastando realizar algumas adaptações no texto, levando em conta o contexto atual da Política de saúde. Dessa forma, considerou-se que o principal problema com relação à PNEPS não é o conteúdo da política, e sim as dificuldades enfrentadas no processo de implementação, que contribuem, inclusive, para a existência de grande heterogeneidade e desigualdade na situação verificada em cada unidade federada.

Os debates, assim, confluíram para a identificação de um conjunto de problemas e elaboração de propostas e recomendações com vistas à implementação, execução, gestão, monitoramento e avaliação da PNEPS, as quais foram agrupadas, de acordo com as diferentes dimensões da política, em seis categorias: a) Conceito de EPS; b) Gestão de recursos financeiros; c) Institucionalização da política; d) Descentralização e regionalização do processo de implementação da PNEPS; e) Monitoramento e avaliação da Política; f) Relação/articulação entre entes federativos no processo de implementação da PNEPS. A seguir, apresentamos uma síntese dos problemas identificados e das propostas elaboradas nas Oficinas em cada uma dessas dimensões.

\section{Problemas identificados nas Oficinas Regionais}

Com base no processamento das informações extraídas dos relatórios das Oficinas Regionais, foi construída uma matriz de análise que sistematiza os principais problemas enfrentados no 
processo de implementação da PNEPS. Apesar da heterogeneidade constatada em termos do grau de desenvolvimento da gestão, planejamento, programação e execução das ações de EPS, podem-se identificar 'áreas-problema', que se apresentam, em maior ou menor grau, em todos os estados. São elas:

Gestão da Política de EPS: constatou-se a complexidade do processo de gestão da implementação da PNEPS, devido a uma série de fatores; entre os quais, destaca-se a falta de compreensão, por parte da maioria dos gestores, da importância das ações de EPS para a qualificação da gestão e da melhoria da atenção à saúde no SUS. Desse fato, mais evidente em alguns estados, deriva a falta de priorização das ações de EPS no processo de planejamento e programação, quer seja no âmbito do Plano Estadual de Saúde, quer, mais precisamente, na fragilidade dos processos de planejamento regional das ações de EPS. Isso também está conectado com o grau de desenvolvimento do processo de implantação e funcionamento das Cies, e se reflete na articulação entre as diversas instituições que compõem o quadrilátero da EPS ${ }^{23}$. Alguns estados apontam dificuldades na relação com as instituições de ensino, bem como a incipiente incorporação dos representantes dos trabalhadores de saúde e movimentos sociais no processo de planejamento e implementação da Política e dos Planos de EPS, além de apontarem, em alguns casos, a grande rotatividade dos gestores, especialmente ao nível municipal. Outro aspecto discutido durante as Oficinas foi a necessidade de aprofundar o papel que pode ser representado pelos Contratos Organizativos de Ação Pública Ensino-Saúde (Coapes) na organização das ações de EPS e na articulação ensino-serviço, inclusive na negociação e nas relações entre as instituições públicas e privadas.

Financiamento: todos os estados apontaram a descontinuidade dos repasses financeiros do MS (desde 2011) e problemas na gestão dos recursos, especialmente a dificuldade de remunerar docentes vinculados às escolas do SUS, assim como da utilização dos recursos em função das restrições existentes na legislação pública nessa área. Alguns estados têm buscado alternativas para garantir o financiamento dessas ações, a exemplo de Santa Catarina, Ceará e outros, disponibilizando recursos de outras fontes, inclusive do orçamento estadual.

Modelo de formação/educação permanente implementado: os participantes das Oficinas apontaram a coexistência de um modelo de capacitação tradicional, caracterizado pela reprodução de cursos e atividades voltadas a categorias profissionais isoladas, com a implantação de um modelo inovador, que leva em conta o trabalho em equipe, a educação interprofissional, a utilização de metodologias ativas de ensino-aprendizagem e a realização de atividades de educação a distância com uso de Tecnologias de Informação Comunicação (Tics) e a demanda por formação em metodologias ativas e ações inovadoras para o desenvolvimento da área de EPS.

Infraestrutura: alguns estados referem as deficiências de espaço físico e apoio logístico para garantir o funcionamento das Cies, como também apontam a inexistência do setor de EPS no organograma da SES, além da falta de recursos (pessoal, diárias, transporte) para a realização das ações de EPS. Também foi referida a inexistência de escolas estaduais de saúde pública em alguns estados.

Conceito de educação permanente: vários representantes dos estados apontaram a necessidade de retomar a reflexão e o debate sobre o conceito de educação permanente, uma vez que se considera não ter havido uma apropriação plena da concepção que fundamenta a PNEPS. Aponta-se, como determinantes desse fato, a instabilidade institucional, especialmente a rotatividade dos gestores, propondo-se certo 'alinhamento conceitual' que facilite o processo de gestão e planejamento das ações de EPS. Nessa perspectiva, destaca-se a necessidade de efetuar a distinção entre 'educação em saúde', 'educação permanente', 'educação popular', distinguindo-se, também, a 'educação profissional' da educação permanente.

Monitoramento e avaliação das atividades de educação permanente: praticamente todos os participantes apontaram dificuldades em 
monitorar e avaliar as ações de educação permanente, enfatizando a ausência de indicadores que superem a mera quantificação dos cursos e outras atividades realizadas.

Atribuições e responsabilidades de cada nível de governo e articulação interfederativa: os representantes dos estados foram unânimes em apontar que, além da descontinuidade dos repasses financeiros, a necessidade do apoio técnico da SGTES para implementação da PNEPS.

\section{Propostas elaboradas nas Oficinas Regionais}

\author{
A identificação dos problemas subsidiou \\ a elaboração de um conjunto de propostas \\ em cada uma das Oficinas, as quais foram \\ sistematizadas em uma matriz (quadro 2), \\ com base na semelhança e aproximação \\ temática.
}

Quadro 2. Propostas elaboradas nas oficinas regionais: síntese

\begin{tabular}{|c|c|}
\hline Área-problema & ropostas \\
\hline $\begin{array}{l}\text { Gestão da Políti- } \\
\text { ca de EPS }\end{array}$ & $\begin{array}{l}\text { - Apoio do MS para fomentar a elaboração dos Planos estaduais de EPS e de Planos de ação regional } \\
\text { - Vincular instrumentos de gestão e planejamento do SUS à EPS } \\
\text { - Implantar sistema de informação/gerenciamento das ações de EPS } \\
\text { - Criar a função de apoiadores regionais na área de EPS } \\
\text { - Incluir e articular as ações de formação promovidas pelo Ministério da Saúde na PNEPS, por exem- } \\
\text { plo: Coapes, Programa de Formação de Profissionais de Nível Médio para a Saúde (Profaps) etc. }\end{array}$ \\
\hline Financiamento & $\begin{array}{l}\text { - Retorno do financiamento federal para accões de EPS } \\
\text { - Garantir participação dos Estados e Municípios no financiamento das aç̃̃es de educação permanente } \\
\text { - Discutir e apresentar planos para viabilizar a execução dos recursos } \\
\text { - Garantir recursos para custeio e investimento para as ações de educação permanente } \\
\text { - Garantir no Plano Plurianual/Lei Orçamentária Anual (PPA/LOA) o repasse orçamentário e finan- } \\
\text { ceiro de um percentual mínimo a ser pactuado, exclusivo para execução das ações da política de } \\
\text { EPS, por parte da União, Estados e Municípios }\end{array}$ \\
\hline & $\begin{array}{l}\text { - Apoiar a reflexão sobre métodos inovadores de ensino } \\
\text { - Vincular ofertas pedagógicas às necessidades de formação } \\
\text { - Realizar seminários a partir de experiências exitosas } \\
\text { - Articular melhor as instituições de ensino no processo de implementação da PNEPS. Pensar } \\
\text { mecanismos para reforçar a participação da IES dentro da Cies } \\
\text { - Estabelecer estratégias para que gestores e instituiç̃̃es de ensino se comprometam em construir } \\
\text { e participar efetivamente das ações de EPS } \\
\text { - Reconhecer a preceptoria nos processos de progressão funcional dos servidores } \\
\text { - Definir as contrapartidas na articulação ensino serviço. } \\
\text { - Qualificar processos/desenhos estratégicos pedagógicos voltadas para problematização, } \\
\text { transformação da realidade e qualificação para o SUS } \\
\text { - Implantar sistema de regulação das práticas de formação e EPS nos serviços }\end{array}$ \\
\hline $\begin{array}{l}\text { Infraestrutura da } \\
\text { Cies }\end{array}$ & $\begin{array}{l}\text { - Estimular a criação de espaços nas Secretarias Estaduais e Municipais para a EPS e Cies } \\
\text { - Garantir que o setor responsável pela Educação Permanente em Saúde (EPS) esteja presente no organo- } \\
\text { grama oficial da Secretaria Estadual de Saúde (SES) e que disponha de estrutura física própria } \\
\text { - Fortalecer as Regiões de Saúde através das Cies }\end{array}$ \\
\hline Conceito EPS & $\begin{array}{l}\text { - Promover o alinhamento conceitual sobre a PNEPS, especialmente a concepção de EPS } \\
\text { - Realizar oficinas de trabalho com gestores e técnicos para alinhamento conceitual em torno da EPS } \\
\text { - Fortalecer as equipes das Escolas do SUS e membros da Cies para facilitarem a discussão sobre o } \\
\text { conceito de EPS } \\
\text { - Incluir a discussão sobre o conceito de EPS nas Comissões Intergestores Regionais (CIR) }\end{array}$ \\
\hline $\begin{array}{l}\text { Monitoramento } \\
\text { e avaliação das } \\
\text { ações de EP }\end{array}$ & $\begin{array}{l}\text { - Estabelecer mecanismos de monitoramento e avaliação da execução dos planos estaduais e regio- } \\
\text { nais de EPS com indicadores de processo e resultados } \\
\text { - Estimular que as áreas técnicas registrem e divulguem as ações de EPS } \\
\text { - Dimensionar/mapear os pontos de educação da rede estadual }\end{array}$ \\
\hline
\end{tabular}




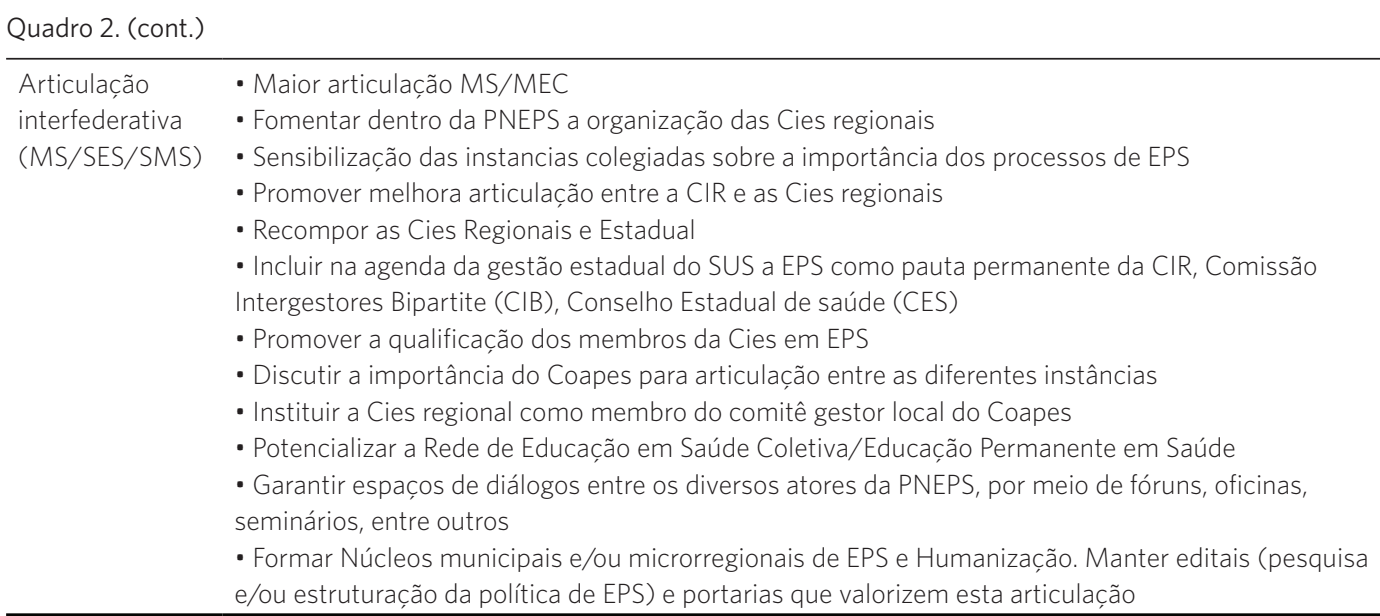

\section{Ações desencadeadas pelo Ministério da Saúde em 2017-2018 para a implementação e fortalecimento da PNEPS}

A partir dos resultados obtidos com a realização das Oficinas Regionais, o Deges/SGTES desencadeou, durante o ano de 2018, uma série de atividades voltadas ao atendimento das demandas dos estados, buscando dar respostas imediatas a alguns dos principais problemas identificados.

Em primeiro lugar, tratou-se de recuperar o fluxo de financiamento, por meio de repasses de recursos do MS aos estados e municípios Tal decisão materializou-se na publicação da Portaria $n^{0} 3.194$, de 28 de novembro de 201724, que dispõe sobre o Programa para o Fortalecimento das Práticas de Educação Permanente em Saúde no Sistema Único de Saúde (Pro EPS-SUS) e cria incentivos financeiros de custeio para implementação, execução e gestão do Pro EPS-SUS, com vistas a estimular, acompanhar e fortalecer a qualificação profissional dos trabalhadores da área para a transformação das práticas de saúde em direção ao atendimento dos princípios fundamentais do SUS, a partir da realidade local e da análise coletiva dos processos de trabalho.
Com isso, foram repassados aos municípios e estados aproximadamente de $\mathrm{R} \$ 70$ milhões para o planejamento e execução de ações educativas que respondessem às necessidades de saúde, respeitando a realidade regional/local.

Paralelamente, tratou-se de incentivar implementação dos processos de integração ensino-serviço-comunidade, ampliando o canal de diálogo com os atores envolvidos com os Coapes. Nesse sentido, como estratégia para alavancar o processo de contratualização no País, investimentos foram feitos para qualificar o processo de negociação permanente em defesa da qualificação do cuidado e da formação.

Buscando dar visibilidade à experiências inovadoras, o Deges/SGTES/MS, em parceria com a Opas/Organização Mundial da Saúde (OMS), lançou o Edital do Laboratório de Inovações em Educação na Saúde com ênfase na Educação Permanente em Saúde, tendo recebido 251 inscrições, que passaram por uma comissão de avaliadores, resultando em uma primeira seleção de 45 experiências que foram apresentadas em seminário nacional. Destas, 30 foram selecionadas para visitação in loco, tendo sido premiadas 15 finalistas, que compuseram uma publicação específica do MS/Opas ${ }^{25}$, organizada em três eixos temáticos: Integração EnsinoServiço-Comunidade; Educação e Práticas 
Interprofissionais; e Gestão da Política de Educação Permanente em Saúde.

Para subsidiar tecnicamente o processo de planejamento e programação das ações de EPS nos estados, foi elaborado um manual, intitulado 'Orientações para o planejamento das ações de Educação Permanente em Saúde no SUS', encaminhado a todas as SES e Secretarias Municipais de Saúde (SMS) para subsidiar a elaboração dos Planos de Educação Permanente.

Também foi organizada uma publicação específica intitulada 'Política Nacional de Educação Permanente em Saúde: o que se tem produzido para o seu fortalecimento?'26 estruturada em quatro capítulos, e que aborda aspectos relevantes do atual movimento em torno da implementação da PNEPS, como integração ensino-serviço; Coapes; programa para fortalecimento da EPS; reconhecimento de experiências de EPS; incorporação de novas abordagens nos processos de EPS, como a educação interprofissional em saúde e incentivo ao planejamento ascendente, participativo e regionalizado das ações de EPS nos estados.

Finalmente, em novembro de 2018, foi realizada, em Brasília, a Oficina Nacional, reunindo cerca de 120 participantes, para validação do Relatório Final das Oficinas Regionais e apresentação da proposta preliminar relativa ao sistema de monitoramento e avaliação das ações de EPS, que será desenvolvido no próximo ano.

\section{Considerações finais}

A realização de um debate nacional sobre a PNEPS possibilitou traçar um panorama dos principais aspectos do processo de implementação dessa política nos estados e municípios do País. Nessa perspectiva, considerou-se necessário identificar as fragilidades enfrentadas em cada realidade concreta, de modo a avançar com a elaboração de proposições, tanto em relação a aspectos gerais da Política e do papel a ser desempenhado por cada nível de governo quanto em relação à elaboração de proposições específicas que possam servir de referência para o aperfeiçoamento do processo de implementação em cada estado.

Desse modo, buscou-se resgatar o protagonismo de quem executa a Política de Educação Permanente, especialmente as Secretarias Estaduais e Municipais de Saúde, em um processo que contou com apoio institucional do MS para o desencadeamento de iniciativas que possibilitaram a retomada do planejamento ascendente, em um esforço coletivo para realização de ações articuladas entre os três entes da federação, valorização das experiências em curso no país, divulgadas por meio do Laboratório de Inovações em Educação na Saúde, as quais evidenciam a potencialidade da PNEPS no que diz respeito à contribuição para a melhoria da qualidade dos serviços prestados pelo SUS.

\section{Colaboradores}

Gonçalves CB (0000-0002-7644-9440)* participou substancialmente da concepção, planejamento, análise e interpretação dos dados, participou significativamente na revisão crítica do conteúdo e participou da aprovação da versão final do manuscrito. Pinto ICM (0000-0002-1636-2909)* participou substancialmente da concepção, planejamento, análise e interpretação dos dados e participou significativamente na elaboração do rascunho e da revisão crítica do conteúdo. França T (0000-0002-8209-9811)* e Teixeira CF (0000-0002-8080-9146)* participaram substancialmente da concepção, planejamento, análise e interpretação dos dados, participaram significativamente na revisão crítica do conteúdo e participou da aprovação da versão final do manuscrito.
*Orcid (Open Researcher and Contributor ID). 


\section{Referências}

1. World Health Organization. WHO Health Work Force 2030: towards a global strategy on human resources for health world Health Organization; 2015.

2. Relatório da 29 Conferencia Sanitaria Panamericana. 69 Sessão do Comitê Regional da OMS para as Américas; 25-29 set. 2017; Washington, (DC): Organização PanAmericana de Saúde. (OMS/OPAS); 2017.

3. Teixeira CFS, Souza LEP, Paim JS. Sistema Único de Saúde (SUS): a difícil construção de um sistema universal na sociedade brasileira. In: Paim JS, Almeida Filho N. Saúde Coletiva: teoria e prática. Rio de Janeiro: MedBook; 2014.

4. Viacava F, Oliveira RAD, Carvalho C, et al. SUS: oferta, acesso e utilização de serviços de saúde nos últimos 30 anos. Ciênc. Saúde Colet. 2018; 23(6):1751-1762.

5. Machado MH, Ximenes Neto FRG. Gestão da Educação e do Trabalho em Saúde no SUS: trinta anos de avanços e desafios. Ciênc. Saúde Colet. 2018; 23(6):1971-1979.

6. Noronha JC, Noronha GS, Pereira TR, et al. Notas sobre o futuro do SUS: breve exame de caminhos e descaminhos trilhados em um horizonte de incertezas e desalentos. Ciênc. Saúde Colet. 2018; 23(6):2015-2060

7. Brasil. Portaria GM/MS no 198 de 13 de fevereiro de 2004. Institui a Política Nacional de Educação Permanente em Saúde como estratégia do Sistema Único de Saúde para a formação e o desenvolvimento de trabalhadores para o setor e dá outras providências. Diário Oficial da União. 14 Fev 2004.

8. Brasil. Portaria GM/MS n ${ }^{\circ} 1.996$ de 20 de agosto de 2007. Dispõe sobre as diretrizes para a implementação da Política Nacional de Educação Permanente em Saúde. Diário Oficial da União. 20 Ago 2007.

9. Brasil. Portaria de Consolidação $n^{\circ} 3 \mathrm{GM} / \mathrm{MS}$ de 28 de setembro de 2017. Consolidação das normas sobre as redes do Sistema Único de Saúde. Diário Oficial da União. 29 Set 2017.
10. Ferraz F, Backes VMS, Mercado-Martines FJ, et al. Gestão de recursos financeiros da educação permanente em saúde: desafio das comissões de integração ensino serviço. Ciênc. Saúde Colet. 2013; 18(6):1683-1693.

11. Ferraz L, Vendruscolo C, Marmett S. Educação Permanente na Enfermagem: uma revisão integrativa. Baiana de Enferm. 2014; 28(2):196-207.

12. Nicoletto SCS, Bueno VLRC, Nunes EFPA, et al. Desafios na implantação, desenvolvimento e sustentabilidade da Política de Educação Permanente em Saúde no Paraná, Brasil. Saúde Soc. 2013; 22(4):1094-1105.

13. Macedo NB, Albuquerque PC, Medeiros KR. O desafio da implementação da educação permanente na gestão da educação na saúde. Trab. Educ. Saúde. 2014; 12(2):379-401.

14. França T, Pierantoni C, Belisário AS, et al. A capilaridade da Política Nacional de Educação Permanente em Saúde no Brasil. Atas-Investig. Qualit. Saúde. 2016; 2:66-75.

15. Paim JS. Financiamento da Saúde: questão política para além do setor. Cremeb-Vida \& Ética. 2014; 5(18):17.

16. Vieira FS. Nota Técnica. Crise econômica, austeridade fiscal e saúde: que lições podem ser aprendidas? Ipea: Brasília, DF; 2016.

17. Scheffer M. O capital estrangeiro e a privatização do sistema de saúde brasileiro. Cad. Saúde Pública. 2015; 31(4):663-666.

18. Kingdon, J. Agendas, alternatives and public choices. Boston: Little Brow; 1994.

19. Pinto ICM, Vieira-da-Silva LM, Baptista TVF. Ciclo de uma política pública de saúde: problematização, construção da agenda, institucionalização, formulação, implementação e avaliação. In: Paim JS, Almeida Filho N. Saúde Coletiva: teoria e prática. Rio de Janeiro: MedBook; 2014. 
20. Brasil. Lei n 8.080 de 19 de setembro de 1990. Dispõe sobre as condições para a promoção, proteção e recuperação da saúde, a organização e o funcionamento dos serviços correspondentes e dá outras providências. Diário Oficial da União. 20 Set 1990.

21. Brasil. Ministério da Saúde Relatório Consolidado sobre o processo de implementação da Política Nacional de Educação permanente em Saúde (PNEPS). SGTES/DEGES/Ministério da Saúde: Brasília, DF; 2018.

22. Brasil. Ministério da Saúde. Termo de referência para as Oficinas Regionais PNEPS. SGTES/DEGES/ Ministério da Saúde: Brasília, DF; 2017.

23. Ceccim RB, Feuerwerker LCM. O Quadrilátero da formação para a área da saúde: ensino, gestão, formação e controle social. Physis. 2014; 14(1):41-65.
24. Brasil. Ministério da Saúde. Portaria no 3.194, de 28 de novembro de 2017. Dispõe sobre o Programa para o Fortalecimento das Práticas de Educação Permanente em Saúde no Sistema Único de Saúde - PRO EPS-SUS. Diário Oficial da União. 29 Nov 1990.

25. Brasil. Ministério da Saúde. Laboratório de Inovação em Educação na Saúde com ênfase em Educação Permanente. Ministério da Saúde/Organização Panamericana da Saúde/Organização Mundial Saúde no Brasil: Brasília, DF; 2018.

26. Brasil. Ministério da Saúde. Política Nacional de Educação Permanente em Saúde: o que se tem produzido para o seu fortalecimento? Ministério da Saúde: Brasília, DF; 2018.

Recebido em 14/12/2018

Aprovado em 18/04/2019

Conflito de interesses: inexistente

Suporte financeiro: não houve 